RESIDENT

\& FELLOW

SECTION

Section Editor

Mitchell S.V. Elkind,

MD, MS

Alexandra Borchert, MD

Gabriel Möddel, MD

Matthias Schilling, MD

Address correspondence and reprint requests to Dr. Matthias Schilling, Dept. of Neurology, Universitätsklinikum Münster, Albert-Schweitzer-Str. 33, D-48129 Münster, Germany schillim@uni-muenster.de

Supplemental data at www.neurology.org

\title{
Teaching Video NeuroImages: Paroxysmal kinesigenic dyskinesia
}

向

A man presented multifocal brachiofacial or generalized dystonic movements and dyskinesias (video on the Neurology ${ }^{\circledR}$ Web site at www.neurology.org). Episodes started with a variable aura, triggered by emotional stress or movement initiation. Clinical examination and diagnostics including brain MRI and EEG were normal. Attacks stopped after therapy with carbamazepine, $200 \mathrm{mg}$ daily, was started.

Paroxysmal dyskinesia is a rare, mostly autosomal dominant disorder characterized by kinesigenic triggers, attacks $<1$ minute, preserved consciousness, absence of structural diseases or epileptiform activity, and effect of phenytoin or carbamazepine. ${ }^{1,2} \mathrm{Al}$ though the term kinesigenic dyskinesia is used, other triggers are possible. The movements often include dystonic postures, with or without dyskinesias.

\section{REFERENCES}

1. Houser MK, Soland VL, Bhatia KP, et al. Paroxysmal kinesigenic choreoathetosis: a report of 26 patients. J Neurol 1996;246:120-126.

2. Bruno MK, Hallett M, Gwinn-Hardy K, et al. Clinical evaluation of idiopathic paroxysmal kinesigenic dyskinesia: new diagnostic criteria. Neurology 2004;63:2280-2287. 


\title{
Neurology
}

\author{
Teaching Video NeuroImages: Paroxysmal kinesigenic dyskinesia \\ Alexandra Borchert, Gabriel Möddel and Matthias Schilling \\ Neurology 2009;72;e118 \\ DOI 10.1212/WNL.0b013e3181a92b66
}

This information is current as of June 8, 2009

\section{Updated Information \& Services \\ Supplementary Material \\ References \\ Subspecialty Collections}

Permissions \& Licensing

Reprints including high resolution figures, can be found at: http://n.neurology.org/content/72/23/e118.full

Supplementary material can be found at: http://n.neurology.org/content/supp1/2009/06/07/72.23.e118.DC1

This article cites 2 articles, 1 of which you can access for free at: http://n.neurology.org/content/72/23/e118.full\#ref-list-1

This article, along with others on similar topics, appears in the following collection(s):

All Clinical Neurology

http://n.neurology.org/cgi/collection/all_clinical_neurology

All Movement Disorders

http://n.neurology.org/cgi/collection/all_movement_disorders

Dystonia

http://n.neurology.org/cgi/collection/dystonia

Nonepileptic seizures

http://n.neurology.org/cgi/collection/nonepileptic_seizures

Information about reproducing this article in parts (figures,tables) or in its entirety can be found online at:

http://www.neurology.org/about/about_the_journal\#permissions

Information about ordering reprints can be found online:

http://n.neurology.org/subscribers/advertise

Neurology ${ }^{\circledR}$ is the official journal of the American Academy of Neurology. Published continuously since 1951, it is now a weekly with 48 issues per year. Copyright. All rights reserved. Print ISSN: 0028-3878. Online ISSN: 1526-632X.

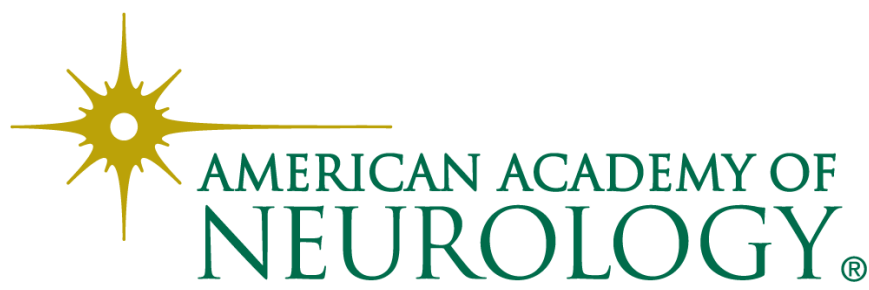

